ANNALES

POLONICI MATHEMATICI

LXXVIII.3 (2002)

\title{
Laurent series expansion for solutions of hypoelliptic equations
}

\author{
M. LANGEnBRUCH (Oldenburg)
}

\begin{abstract}
We prove that any zero solution of a hypoelliptic partial differential operator can be expanded in a generalized Laurent series near a point singularity if and only if the operator is semielliptic. Moreover, the coefficients may be calculated by means of a Cauchy integral type formula. In particular, we obtain explicit expansions for the solutions of the heat equation near a point singularity. To prove the necessity of semiellipticity, we additionally assume that the index of hypoellipticity with respect to some variable is 1 .
\end{abstract}

This paper is concerned with expansions of zero solutions of hypoelliptic partial differential operators near isolated singularities. For holomorphic functions, such an expansion is provided by the well known Laurent series. This has been extended to zero solutions of elliptic partial differential operators by several authors (see Harvey and Polking [3], Wachman [15]; for solutions of elliptic systems see Tarkhanov $[12,13])$. For solutions of hypoelliptic operators, such expansions apparently are not known (see Ligocka [10, Problem 4.5]), and they will be discussed in the present paper.

To be more specific, we start with an appropriate extension of the Laurent series expansion for holomorphic functions to the case of solutions of partial differential operators (see Hörmander [4] and the next section for the relevant notations):

Let $P(D)$ be a hypoelliptic partial differential operator with constant coefficients in $n$ variables and with $\operatorname{deg} P=: m$. Let

$$
C_{P}^{\infty}(U):=\left\{f \in C^{\infty}(U) \mid P(D) f=0\right\}
$$

denote the zero solutions of $P(D)$ on an open set $U \subset \mathbb{R}^{n}$. Without loss of generality we can assume that the canonical $n$th unit vector is noncharacteristic for $P(D)$. We fix an elementary solution $E$ for $P(D)$ and consider

2000 Mathematics Subject Classification: Primary 35H10, 35C10; Secondary 35E20.

Key words and phrases: Laurent series, isolated singularities, semielliptic operators, heat equation. 
series of the form

$$
\sum_{a \in I} c_{a} \partial^{a} E
$$

where $c_{\alpha} \in \mathbb{C}$ and

$$
I:=\left\{a \in \mathbb{N}_{0}^{n} \mid 0 \leq a_{n} \leq m-1\right\} .
$$

(0.1) can be considered as a (generalized) Laurent series for solutions of $P(D)$ : in fact, in the case of holomorphic functions, $P(D)$ is the Cauchy-Riemann operator $\bar{\partial}:=\frac{1}{2}\left(\partial_{1}+i \partial_{2}\right), m:=\operatorname{deg} P=1$ and $I=\left\{(a, 0) \mid a \in \mathbb{N}_{0}\right\}$. We can take the canonical elementary solution $E(x):=1 /\left(\pi\left(x_{1}+i x_{2}\right)\right)$ for $\bar{\partial}$ and (0.1) then turns into

$$
\sum_{k=-\infty}^{-1} c_{k}\left(x_{1}+i x_{2}\right)^{k}
$$

which is the principal part of a general Laurent series.

Let $\varrho=\left(\varrho_{1}, \ldots, \varrho_{n}\right) \in\left[1, \infty\left[^{n}\right.\right.$ be the index of hypoellipticity of $P(D)$. Then $\left.E\right|_{\mathbb{R}^{n} \backslash\{0\}}$ is in the Gevrey class $\Gamma^{\varrho}\left(\mathbb{R}^{n} \backslash\{0\}\right)$, and therefore, the series (0.1) uniformly converges on compact subsets of $\mathbb{R}^{n} \backslash\{0\}$ if

$$
\left(c_{a}\right)_{a \in I} \in \Lambda_{I}(\varrho):=\left\{\left(c_{a}\right)_{a \in I}\left|\sup _{a \in I}\right| c_{a} \mid C^{|a|} a^{\varrho a}<\infty \text { for any } C \geq 1\right\} .
$$

In the case of holomorphic functions, $\varrho=(1,1)$ since $\bar{\partial}$ is elliptic. $(0.3)$ then just means that in $(0.2), c_{k}=O\left(\varepsilon^{k} / k !\right)$ for any $\varepsilon>0$, which is equivalent to the convergence of $(0.2)$ on $\mathbb{R}^{2} \backslash\{0\}$.

An appropriate version of a theorem on Laurent series expansion for zero solutions of $P(D)$ near an isolated singularity is thus provided by the following property $(P)$ : Let $I$ and $\varrho$ be as above. Let $U \subset \mathbb{R}^{n}$ be open and $0 \in U$.

$(P) \quad$ For any $f \in C_{P}^{\infty}(U \backslash\{0\})$, there are $\left(c_{a}\right)_{a \in I} \in \Lambda_{I}(\varrho)$ and $g \in C_{P}^{\infty}(U)$ such that

$$
f=\sum_{a \in I} c_{a} \partial^{a} E+\left.g\right|_{U \backslash\{0\}} .
$$

Let $\varrho_{n}=1$. We will show that then $P(D)$ satisfies $(P)$ for some $U$ iff $P(D)$ satisfies $(P)$ for any $U$ iff $P(D)$ is semielliptic. Moreover, the coefficients $\left(c_{a}\right)_{a \in I}$ can be calculated from $f$ by means of a generalized Cauchy type integral formula (see Remark 2.1).

The proofs are based on the results of Langenbruch [7, 8], especially on a Grothendieck type duality for zero solutions of hypoelliptic operators.

We finally discuss the classification of poles and removable singularities for solutions of hypoelliptic equations. 
1. Preliminaries. In this section, some material is provided which is needed to prove the main results in Section 2.

In this paper, $P(D)$ always denotes a partial differential operator with constant coefficients in $n$ variables. Let $C_{P}^{\infty}(U)$ denote the $C^{\infty}$ zero solutions of $P(D)$ on $U$.

For a compact set $K \subset U$, the quotient space $C_{P}^{\infty}(U \backslash K) / C_{P}^{\infty}(U)$ may be considered as the space of singularities on $K$ of zero solutions of $P(D)$. We first show that this space is independent of $U$. As a consequence, property $(P)$ from our introduction is independent of $U$ (see Corollary 1.2).

1.1. REMARK. Let $K \subset U$ be compact. The canonical mapping

$$
i: B V_{K}(P):=C_{P}^{\infty}\left(\mathbb{R}^{n} \backslash K\right) / C_{P}^{\infty}\left(\mathbb{R}^{n}\right) \rightarrow C_{P}^{\infty}(U \backslash K) / C_{P}^{\infty}(U)
$$

is an isomorphism.

Proof. Obviously, $i$ is well defined and injective. Let $g \in C_{P}^{\infty}(U \backslash K)$. Choose $\varphi \in C_{0}^{\infty}(U)$ such that $\varphi=1$ near $K$. Then $P(D)(\varphi g)$ can trivially be extended to a function $h \in C_{0}^{\infty}(U \backslash K)$. Let $E$ be a distributional elementary solution for $P(D)$. Then $f:=g \varphi-E * h \in C^{\infty}\left(\mathbb{R}^{n} \backslash K\right)$ and

$$
P(D) f=P(D)(g \varphi)-h=0 \quad \text { on } \mathbb{R}^{n} \backslash K
$$

by the definition of $h$. Similarly, $f-g=g(\varphi-1)-E * h \in C^{\infty}(U)$ and

$$
P(D)(f-g)=0 \quad \text { on } U \text {. }
$$

Thus, $i([f])=[g]$, and $i$ is surjective.

Let $\varrho$ be the index of hypoellipticity of $P(D)$ (see Hörmander [4, Section 11]) and let

$$
\Gamma^{\varrho}(U):=\left\{f \in C^{\infty}(U) \mid \forall K \subset \subset U \exists C \geq 1: \sup _{x \in K, a \in \mathbb{N}_{0}^{n}} \frac{\left|f^{(a)}(x)\right|}{C^{|a|} a^{\varrho a}}<\infty\right\} .
$$

1.2. Corollary. The following are equivalent:

(i) $P(D)$ satisfies $(P)$ for some $U$.

(ii) $P(D)$ satisfies $(P)$ for any $U$.

(iii) $P(D)$ satisfies $(P)$ for $U:=\mathbb{R}^{n}$.

Proof. (i) $\Rightarrow$ (iii). Let $f \in C_{P}^{\infty}\left(\mathbb{R}^{n} \backslash\{0\}\right)$ and choose $\left(c_{a}\right)_{a \in I}$ and $g \in$ $C_{P}^{\infty}(U)$ for $\left.f\right|_{U \backslash\{0\}}$ by $(P)$ for $U$. Since $E \in \Gamma^{\varrho}\left(\mathbb{R}^{n} \backslash\{0\}\right.$ ) (by Hörmander [4, Theorem 11.4.12]) and $\left(c_{a}\right)_{a \in I} \in \Lambda_{I}(\varrho)$ (see (0.3)), the series $\sum_{a \in I} c_{a} \partial^{a} E$ converges on $\mathbb{R}^{n} \backslash\{0\}$. Since

$$
\widetilde{g}:=f-\sum_{a \in I} c_{a} \partial^{a} E \in C_{P}^{\infty}\left(\mathbb{R}^{n} \backslash\{0\}\right) \quad \text { and }\left.\quad \widetilde{g}\right|_{U \backslash\{0\}}=\left.g\right|_{U \backslash\{0\}}
$$

by $(P)$ for $U$, we have $\widetilde{g} \in C_{P}^{\infty}\left(\mathbb{R}^{n}\right)$.

(iii) $\Rightarrow$ (ii). Let $f \in C_{P}^{\infty}(U \backslash\{0\})$. Choose $\tilde{f} \in C_{P}^{\infty}\left(\mathbb{R}^{n} \backslash\{0\}\right)$ and $g_{1} \in$ $C_{P}^{\infty}(U)$ by Remark 1.1 such that $f=\left.\left(\tilde{f}+g_{1}\right)\right|_{U \backslash\{0\}}$. With $\left(c_{a}\right)_{a \in I} \in \Lambda_{I}(\varrho)$ 
and $g \in C_{P}^{\infty}\left(\mathbb{R}^{n}\right)$ chosen for $\tilde{f}$ by $(P)$ for $\mathbb{R}^{n}$, we get

$$
f=\sum_{a \in I} c_{a} \partial^{a} E+\left.\left(g_{1}+g\right)\right|_{U \backslash\{0\}} .
$$

This proves $(P)$ for $U$.

We now briefly introduce the duality theory for germs of zero solutions of hypoelliptic operators (see Langenbruch [8] for more details): Let $K \subset \mathbb{R}^{n}$ be compact. The space of germs of $C^{\infty}$ zero solutions of $P(D)$ near $K$ is

$$
C_{P}^{\infty}(K):=\operatorname{limind}_{U \supset \supset K} C_{P}^{\infty}(U) .
$$

It is endowed with the locally convex inductive limit topology $\tau_{K}$. If $P$ is not elliptic, then $\tau_{K}$ is not separated (see Langenbruch [8, Bemerkung 1.1]). Let $N_{P}(K):=\operatorname{ker} \tau_{K}$ and $J_{U, K}: C_{P}^{\infty}(U) \rightarrow C_{P}^{\infty}(K)$ be the canonical mapping. Then $N_{P}(U, K):=\left(J_{U, K}\right)^{-1}\left(N_{P}(K)\right)$ is closed in $C_{P}^{\infty}(U)$ and

$$
\widetilde{C}_{P}^{\infty}(K):=C_{P}^{\infty}(K) / N_{P}(K)=\operatorname{limind}_{U \supset \supset}\left(C_{P}^{\infty}(U) / N_{P}(U, K)\right)
$$

topologically. The canonical (restriction) mappings $\widetilde{J}_{U, V}: C_{P}^{\infty}(U) / N_{P}(U, K)$ $\rightarrow C_{P}^{\infty}(V) / N_{P}(V, K)$ are well defined, compact and injective if $V$ is relatively compact in $U$. Therefore,

$$
\widetilde{C}_{P}^{\infty}(K) \text { is a }(\mathrm{DFS}) \text {-space. }
$$

Let $\check{P}(D):=P(-D)$. A bilinear form $():, C_{P}^{\infty}\left(\mathbb{R}^{n} \backslash K\right) \times C_{\check{P}}^{\infty}(K) \rightarrow \mathbb{C}$ is defined in the following way: Let $g \in C_{\check{P}}^{\infty}(K)$ be defined on $U \supset \supset K$. Choose $\varphi \in C_{0}^{\infty}(U)$ such that $\varphi=1$ near $K$. Then

$$
(f, g):=\langle f, P(-D)(\varphi g)\rangle, \quad f \in C_{P}^{\infty}\left(\mathbb{R}^{n} \backslash K\right),
$$

is defined and is independent of the choice of $\varphi$.

For a locally convex space $E$, let $E_{b}^{\prime}$ denote the dual space endowed with the strong topology. A central tool for the considerations in this paper is provided by the following generalization of the Grothendieck duality:

1.3. TheOREm (Langenbruch [8, Satz 2.4]). Let $P(D)$ be hypoelliptic and let $K \subset \mathbb{R}^{n}$ be compact. The bilinear form (1.2) provides a topological isomorphism between $B V_{K}(P):=C_{P}^{\infty}\left(\mathbb{R}^{n} \backslash K\right) / C_{P}^{\infty}\left(\mathbb{R}^{n}\right)$ and $\left(\widetilde{C}_{\check{P}}^{\infty}(K)\right)_{b}^{\prime}$.

2. Isolated singularities. The main results on Laurent series expansion of zero solutions of hypoelliptic operators are proved in this section. We have seen in Corollary 1.2 that we may restrict our considerations to $f \in C_{P}^{\infty}\left(\mathbb{R}^{n} \backslash\{0\}\right)$. We will first show that such expansions are unique under mild assumptions (see Remark 2.1).

In the following, $P(D)$ will always denote a hypoelliptic operator with $\operatorname{deg} P=: m$ and index of hypoellipticity $\varrho$ such that the canonical $n$th unit 
vector $e_{n}$ is noncharacteristic for $P$. Let

$$
I:=\left\{a \in \mathbb{N}_{0}^{n} \mid 0 \leq a_{n} \leq m-1\right\} .
$$

2.1. REMARK. Let $f \in C_{P}^{\infty}\left(\mathbb{R}^{n} \backslash\{0\}\right)$. Assume that for some compact set $K$ and some open set $U$ with $0 \in K \subset U$, there are $\left(c_{a}\right)_{a \in I} \in \mathbb{C}^{I}, g \in C_{P}^{\infty}(U)$ and elementary solutions $E_{a}$ of $P(D)$ such that

$$
\sum_{a \in I} c_{a} \partial^{a} E_{a} \text { converges in } C(U \backslash K)
$$

and

$$
\left.f\right|_{U \backslash K}=\sum_{a \in I} c_{a} \partial^{a} E_{a}+\left.g\right|_{U \backslash K} .
$$

Then $\left(c_{a}\right)_{a \in I}$ and $g$ are uniquely determined. For $a:=(b, j) \in I$ we have

$$
c_{a}=\left(f, X_{a}\right)
$$

where (, ) is the bilinear form from (1.2) and $X_{a}=X_{(b, j)}$ is the (unique) solution of the Cauchy problem

$$
P(-D) X_{(b, j)}=0 \quad \text { on } \mathbb{R}^{n}, \quad \partial_{n}^{k} X_{(b, j)}\left(x^{\prime}, 0\right)=x^{\prime b} \delta_{k j} / b ! \quad \text { on } \mathbb{R}^{n-1} .
$$

Proof. $X_{(b, j)}$ exists by the Cauchy-Kovalevskaya theorem since $e_{n}$ is noncharacteristic. We choose $\varphi \in C_{0}^{\infty}(U)$ in (1.2) such that $\varphi=1$ near $K$. Since the series in (2.1) converges in $C_{P}^{\infty}(U \backslash K)$, we then have

$$
\begin{aligned}
\left(f, X_{(b, j)}\right) & =\sum_{(d, k) \in I} c_{(d, k)}\left(\partial^{(d, k)} E_{(d, k)}, X_{(b, j)}\right) \\
& =\sum_{(d, k) \in I} c_{(d, k)}\left\langle\partial^{(d, k)} \delta, X_{(b, j)}\right\rangle=c_{(b, j)} .
\end{aligned}
$$

Thus, $\left(c_{a}\right)_{a \in I}$ is uniquely determined, and $g$ is therefore also unique on $U \backslash K$, hence also on $U$, since zero solutions with compact support vanish.

Formula (2.3) can be considered as a generalized Cauchy type integral formula. In fact, the bilinear form ( , ) from (1.2) can also be calculated using $\chi_{V}$, the characteristic function of an open zero neighbourhood $V$, instead of $\varphi \in C_{0}^{\infty}\left(\mathbb{R}^{n}\right)$.

Thus

$$
c_{a}=\left(f, X_{a}\right)=\left\langle f, P(-D)\left(X_{a} \chi_{V}\right)\right\rangle=\sum_{0 \neq b}\left\langle f P^{(b)}(-D) X_{a},(-D)^{b} \chi_{V}\right\rangle / b ! .
$$

$(-D)^{b} \chi_{V}$ is a distribution with support in $\partial V$ and the right-hand side is a sum of integrals over $\partial V$ if $V$ has smooth boundary. In this way, (2.3) provides an explicit integral formula for concrete operators (e.g. the Laplacian or the heat equation), if $V$ is chosen suitably. 
Using $X_{a}$ from (2.4) for $a \in I$, we define the mapping

$$
L: C_{P}^{\infty}\left(\mathbb{R}^{n} \backslash\{0\}\right) \rightarrow \mathbb{C}^{I}, \quad L(f):=\left(\left(f, X_{a}\right)\right)_{a \in I} .
$$

Let

$$
\begin{aligned}
& \Lambda_{I}(\varrho, C):=\left\{\left(c_{a}\right)_{a \in I} \mid\left\|\left(c_{a}\right)\right\|_{C}:=\left(\sum_{a \in I}\left|c_{a}\right|^{2} a^{2 \varrho a} / C^{2|a|}\right)^{1 / 2}<\infty\right\}, \\
& \Gamma_{I}(\varrho, C):=\left\{\left.\left(c_{a}\right)_{a \in I}||\left(c_{a}\right)\right|_{C}:=\left(\sum_{a \in I}\left|c_{a}\right|^{2} C^{2|a|} / a^{2 \varrho a}\right)^{1 / 2}<\infty\right\} .
\end{aligned}
$$

We now prove a useful necessary condition for a weaker form of property $(P)$. Let

$$
R: \widetilde{C}_{P}^{\infty}(\{0\}) \rightarrow \mathbb{C}^{I}, \quad R(f):=\left(f^{(a)}(0)\right)_{a \in I} .
$$

Let $B_{C}:=\left\{x \in \mathbb{R}^{n}|| x \mid \leq C\right\}$.

2.2. TheOREM. Let $P(D)$ have the following property:

$(P 1) \quad$ for any $f \in C_{P}^{\infty}\left(\mathbb{R}^{n} \backslash\{0\}\right)$ there are $C \geq 1,\left(c_{a}\right)_{a \in I}, g \in C_{P}^{\infty}\left(\mathbb{R}^{n}\right)$ and elementary solutions $E_{a}$ of $P(D)$ such that

$$
\left(c_{a}\right)_{a \in I} \in \Lambda_{I}(\varrho, C) \quad \text { and }\left.\quad f\right|_{\mathbb{R}^{n} \backslash B_{C}}=\left.\left(\sum_{a \in I} c_{a} \partial^{a} E_{a}+g\right)\right|_{\mathbb{R}^{n} \backslash B_{C}} .
$$

Then there is $C \geq 1$ such that

$$
L: C_{P}^{\infty}\left(\mathbb{R}^{n} \backslash\{0\}\right) \rightarrow \Lambda_{I}(\varrho, C) \text { is continuous }
$$

and

$$
R \circ{ }^{t} L=\mathrm{id}: \Gamma_{I}(\varrho, C) \rightarrow \mathbb{C}^{I} .
$$

Proof. (a) Since $\left(c_{a}\right)_{a \in I} \in \Lambda_{I}(\varrho, C)$, the sum $\sum_{a \in I} c_{a} \partial^{a} E$ converges on $\mathbb{R}^{n} \backslash B_{C_{1}}$ for some $C_{1} \geq 1$ (see Trèves [14, Chap. 7] or Langenbruch [7, Section 2]).

By $(P 1)$ and Remark 2.1,

$$
L\left(C_{P}^{\infty}\left(\mathbb{R}^{n} \backslash\{0\}\right)\right) \subset \Lambda_{I}(\varrho, \infty):=\operatorname{ind}_{C \rightarrow \infty} \lim _{I}(\varrho, C) .
$$

By the closed graph theorem, $L: C_{P}^{\infty}\left(\mathbb{R}^{n} \backslash\{0\}\right) \rightarrow \Lambda_{I}(\varrho, \infty)$ is continuous. By Grothendieck's lemma (Meise-Vogt $[11,24.33]), L\left(C_{P}^{\infty}\left(\mathbb{R}^{n} \backslash\{0\}\right)\right) \subset \Lambda_{I}(\varrho, C)$ for some $C \geq 1$, and (2.7) follows.

(b) Since $C_{P}^{\infty}\left(\mathbb{R}^{n}\right) \subset \operatorname{ker} L$ and since $\Lambda_{I}(\varrho, C)^{\prime}=\Gamma_{I}(\varrho, C)$, by (1.1) and the duality in Theorem 1.3 we see that ${ }^{t} L: \Gamma_{I}(\varrho, C) \rightarrow \widetilde{C}_{\breve{P}}^{\infty}(\{0\})$ is defined and continuous. Since $R \circ{ }^{t} L$ and id are continuous, (2.8) has to be checked only on the canonical orthonormal basis $\left\{e_{a} \mid a \in I\right\}$. Notice that $R\left(\left[X_{a}\right]\right)=$ $e_{a}$ for $a \in I$. To prove (2.8), it thus suffices to show that

$$
\left(f,{ }^{t} L\left(e_{a}\right)\right)=\left(f, X_{a}\right) \quad \text { for } a \in I \text { and } f \in C_{P}^{\infty}\left(\mathbb{R}^{n} \backslash\{0\}\right) .
$$


This is seen as follows: for $b \in I$ we have

$$
\left(\partial^{b} E,{ }^{t} L\left(e_{a}\right)\right)=\left\langle L\left(\partial^{b} E\right), e_{a}\right\rangle=\left\langle e_{b}, e_{a}\right\rangle=\left(\partial^{b} E, X_{a}\right) .
$$

Thus, (2.9) holds for $f \in F:=\operatorname{span}\left\{\partial^{b} E \mid b \in I\right\}$, and thus for any $f \in$ $C_{P}^{\infty}\left(\mathbb{R}^{n} \backslash\{0\}\right)$ since, by assumption, $f$ is contained in the closure of $F$ in $C_{P}^{\infty}\left(\mathbb{R}^{n} \backslash B_{C}\right)$ for some $C \geq 1$.

By (2.8), ${ }^{t} L\left(\left(c_{a}\right)_{a \in I}\right)$ can be interpreted as the solution of a pointwise Cauchy problem for $P(-D)$ with data in $\Gamma_{I}(\varrho, C)$. Thus $(2.8)$ is an E. Borel type theorem for $C_{\breve{P}}^{\infty}(\{0\})$.

For $\widetilde{m}:=\left(m_{1}, \ldots, m_{n}\right) \in \mathbb{N}^{n}$ and $\alpha \in \mathbb{N}_{0}^{n}$ let $|\alpha: \widetilde{m}|:=\sum \alpha_{k} / m_{k}$. A polynomial $P$ is called semielliptic (with index $\widetilde{m}$ ) if

$$
P(x)=\sum_{|\alpha: \widetilde{m}| \leq 1} b_{\alpha} x^{\alpha} \quad \text { and } \quad P_{\widetilde{m}}(x):=\sum_{|\alpha: \widetilde{m}|=1} b_{\alpha} x^{\alpha} \neq 0 \quad \text { if } 0 \neq x \in \mathbb{R}^{n} .
$$

2.3. Theorem. Let $\varrho_{n}=1$. If $P(D)$ satisfies $(P 1)$, then $P(D)$ is semielliptic.

Proof. (a) Let

$$
P\left(x^{\prime}, x_{n}\right)=\sum_{k \leq m} Q_{k}\left(x^{\prime}\right) x_{n}^{k} \quad \text { and } \quad H_{P}\left(x^{\prime}\right):=\sum_{k<m}\left|Q_{k}\left(x^{\prime}\right)\right|^{1 /(m-k)} .
$$

It is easily seen that

$$
\left|P\left(x^{\prime}, x_{n}\right)\right| \leq C_{1}\left(H_{P}\left(x^{\prime}\right)+\left|x_{n}\right|\right)^{m} .
$$

On the other hand, since $\varrho$ is the index of hypoellipticity of $P$ and $\varrho_{n}=1$, we have

$$
\left(\sum_{k \leq n-1}\left|x_{k}\right|^{1 / \varrho_{k}}+\left|x_{n}\right|\right)^{m}=\left(\sum_{k \leq n}\left|x_{k}\right|^{1 / \varrho_{k}}\right)^{m} \leq C\left(1+\left|P\left(x^{\prime}, x_{n}\right)\right|\right)
$$

(see Hörmander [4, Chap. 11, especially Lemma 11.1.4 and Theorem 11.4.1]).

(b) We have $H_{P}\left(x^{\prime}\right) \leq C_{2}\left(1+\sum_{k \leq n-1}\left|x_{k}\right|^{1 / \varrho_{k}}\right)$ for any $x^{\prime} \in \mathbb{R}^{n-1}$. Indeed, by Langenbruch $[9,(5.9)]$ there is $C_{3} \geq 1$ such that

$$
1 / C_{3} \leq H_{\check{P}}(\xi) / D_{P}(\xi) \leq C_{3} \quad \text { for } \xi \in \mathbb{R}^{n-1},
$$

where $D_{P}(\xi):=\sup \{|\lambda| \mid P(-\xi,-\lambda)=0\}$. To show (b), we thus have to estimate $D_{P}(\xi)$. By $(2.7)$ and Theorem 1.3 , the mapping ${ }^{t} L: \Gamma_{I}(\varrho, C) \rightarrow$ $\widetilde{C}_{\breve{P}}^{\infty}(\{0\})$ is continuous. Let

$$
\Gamma_{B}:=\left\{\left(c_{k}\right) \in \mathbb{C}^{\mathbb{N}}\left|\sup _{k}\right| c_{k} \mid /\left(k ! B^{k}\right)<\infty\right\} .
$$

For $g \in \widetilde{C}_{\breve{P}}^{\infty}(\{0\})$, let $J(g):=\left(\partial_{n}^{k} g(0)\right)_{k \geq 0}$. Since $\varrho_{n}=1$,

$$
J: \widetilde{C}_{\breve{P}}^{\infty}(\{0\}) \rightarrow \Gamma:=\operatorname{ind}_{B \rightarrow \infty} \lim _{B}
$$


is continuous. Hence, $S:=J \circ{ }^{t} L: \Gamma_{I}(\varrho, C) \rightarrow \Gamma$ is continuous, and Grothendieck's lemma (Meise-Vogt [11, 24.33]) implies that for some $B \geq 1$,

$$
S: \Gamma_{I}(\varrho, C) \rightarrow \Gamma_{B} \text { is continuous. }
$$

Let $\xi \in \mathbb{R}^{n-1}$ and choose $\lambda \in \mathbb{C}$ such that $P(-\xi,-\lambda)=0$. Then $g_{(\xi, \lambda)}:=$ $\exp (i\langle(\xi, \lambda), \cdot\rangle) \in C_{\check{P}}^{\infty}\left(\mathbb{R}^{n}\right)$, and $(2.12)$ can be applied to $R\left(g_{(\xi, \lambda)}\right)$. Since $P(-D) g_{(\xi, \lambda)}=0$, for any $j \in \mathbb{N}_{0}$ there are finitely many $d_{a}, a \in I$, such that $\partial_{t}^{j} g_{(\xi, \lambda)}=\sum d_{a} \partial^{a} g_{(\xi, \lambda)}$. Since $R\left({ }^{t} L\left(R\left(g_{(\xi, \lambda)}\right)\right)\right)=R\left(g_{(\xi, \lambda)}\right)$ by (2.8) we deduce that

$$
S\left(R\left(g_{(\xi, \lambda)}\right)\right)=J\left(g_{(\xi, \lambda)}\right) .
$$

The estimate from (2.12) thus implies: there is $C_{4} \geq 1$ such that for any $(\xi, \lambda)$ as above,

$$
\begin{aligned}
& \exp (|\lambda| /(2 B)) \leq 2 \sup \left(|\lambda / B|^{k} / k !\right)=2 \sup \left|\partial_{n}^{k} g_{(\xi, \lambda)}(0)\right| /\left(k ! B^{k}\right) \\
& \leq C_{4}\left(\sum_{a \in I}\left|\partial^{a} g_{(\xi, \lambda)}(0)\right|^{2} C^{2|a|} / a^{2 \varrho a}\right)^{1 / 2} \\
& =C_{4}\left(\sum_{a \in I}\left|(\xi, \lambda)^{a}\right|^{2} C^{2|a|} / a^{2 \varrho a}\right)^{1 / 2} \\
& \leq C_{1}(1+|\lambda|)^{m-1} \exp \left(\sum_{k \leq n-1} C \varrho_{k}\left|\xi_{k}\right|^{1 / \varrho_{k}}\right) .
\end{aligned}
$$

We have thus proved that

$$
D_{P}(\xi) \leq C_{6}\left(1+\sum_{k \leq n-1}\left|\xi_{k}\right|^{1 / \varrho_{k}}\right)
$$

(c) By (2.10), (2.11) and (b) there is $C_{7} \geq 1$ such that for $x \in \mathbb{R}^{n}$,

$$
\begin{aligned}
& \left(\sum_{k \leq n-1}\left|x_{k}\right|^{1 / \varrho_{k}}+\left|x_{n}\right|\right)^{m} / C_{7} \\
& \quad \leq|P(x)| \leq C_{7}\left(\sum_{k \leq n-1}\left|x_{k}\right|^{1 / \varrho_{k}}+\left|x_{n}\right|\right)^{m} \quad \text { if }|x| \geq C_{7} .
\end{aligned}
$$

With fixed $\xi \in \mathbb{R}^{n}$, we now apply this to $\xi_{\lambda}=\left(\lambda^{\varrho_{1}} \xi_{1}, \ldots, \lambda^{\varrho_{n}} \xi_{n}\right)$ for large $\lambda$. By the right-hand side of (2.13),

$$
P(x)=\sum_{|a: \widetilde{m}| \leq 1} c_{a} x^{a}
$$

for $\widetilde{m}=\left(m / \varrho_{1}, \ldots, m / \varrho_{n}\right)$, while the left-hand side of (2.13) implies that

$$
P_{\widetilde{m}}(x) \neq 0 \quad \text { if } 0 \neq x \in \mathbb{R}^{n} \text {. }
$$

$P_{\widetilde{m}}$ also satisfies (2.13). We apply this to the monomials $P_{\widetilde{m}}\left(t e_{k}\right)$ for the canonical unit vectors $e_{k}$, and conclude that $m / \varrho_{k} \in \mathbb{N}$. Thus, $P$ is semielliptic with index $\widetilde{m}$. 
2.4. TheOREM. Let $P$ be semielliptic with index $\widetilde{m}=\left(m_{1}, \ldots, m_{n}\right)$ and let $m_{n}=m$. Then $P(D)$ satisfies $(P)$ for any $U$ with $\left(c_{a}\right)_{a \in I}$ given by $(2.3)$.

Proof. (a) We will show that

$$
R: \widetilde{C}_{P}^{\infty}(\{0\}) \rightarrow \Gamma_{I}(\varrho):=\operatorname{ind}_{C \rightarrow \infty} \lim _{I}(\varrho, C) \text { is bijective, }
$$

where $R$ is given by (2.6). Assuming this for a moment, we conclude that $L \circ{ }^{t} R\left(e_{\alpha}\right)=e_{\alpha}$ for any $\alpha \in I$ (with $L$ defined in (2.5)). Hence range $L \subset$ $\Lambda_{I}(\varrho):=$ proj $\lim _{C \rightarrow 0} \Lambda_{I}(\varrho, C)$, and $L=\left({ }^{t} R\right)^{-1}$, since ${ }^{t} R$ is bijective and $\Lambda_{I}(\varrho)=\Gamma_{I}(\varrho)^{\prime}$. For $f \in C_{P}^{\infty}\left(\mathbb{R}^{n} \backslash\{0\}\right)$, we thus have $\left(c_{a}\right)_{a \in I}:=L(f) \in \Lambda_{I}(\varrho)$. Hence $\sum_{a \in I} c_{a} \partial^{a} E$ converges in $C\left(\mathbb{R}^{n} \backslash\{0\}\right)$ since $E \in \Gamma^{\varrho}\left(\mathbb{R}^{n} \backslash\{0\}\right)$. By Remark 2.1 we have

$$
L\left(f-\sum_{a \in I} c_{a} \partial^{a} E\right)=0
$$

hence $f-\sum_{a \in I} c_{a} \partial^{a} E$ can be extended to a zero solution on $\mathbb{R}^{n}$ since $L=$ $\left({ }^{t} R\right)^{-1}$ is injective. Thus, $(P)$ is proved for $U:=\mathbb{R}^{n}$ and therefore for any $U$ by Corollary 1.2 .

Proof of (2.14). Let $R_{1}$ be the restriction mapping defined by

$$
R_{1}(f):=\left(\partial_{n}^{j} f(, 0)\right)_{j \leq m-1} .
$$

(i) $R_{1}: C_{\breve{P}}^{\infty}(K) \rightarrow\left(\Gamma^{\varrho}(K)\right)^{m}$ is a topological isomorphism. This follows from Langenbruch [7]. In fact, since $\check{P}$ is semielliptic, $\check{P}$ satisfies the assumptions of [7, Satz 4.4] by [7, Bemerkung 3.4(a)] and the claim follows by using also [7, Satz 5.1], since the assumption " $\nu>1$ " is redundant.

(ii) Let $R_{2}(f):=\left(\partial^{b} f(0)\right)_{b \in \mathbb{N}_{0}^{n-1}}$. Then $R_{2}: \Gamma^{\varrho}(\{0\}) \rightarrow \Gamma(\varrho)$ is surjective by E. Borel's theorem in Gevrey classes (this can be proved as in the case of rotationally invariant Gevrey classes; see e.g. Bonet-Meise-Taylor [2]). Since $R=R_{2} \circ R_{1}$ by natural identifications, it is left to show that the kernel $N^{\varrho}(\{0\})$ of the topology of $\Gamma^{\varrho}(\{0\})$ coincides with the kernel of $R_{2}$, i.e. with the functions in $\Gamma^{\varrho}(\{0\})$ which are flat at 0 .

$N^{\varrho}(\{0\})$ is clearly contained in ker $R_{2}$. To show the opposite inclusion, we notice that $\Gamma^{\varrho}(\{0\})_{b}^{\prime}$ is isomorphic to

$$
H^{\varrho}:=\left\{f \in H\left(\mathbb{C}^{n-1}\right)|| f(z) \mid \leq C_{N} \exp \left(\frac{1}{N} v(z)\right) \text { for any } N \geq 1\right\}
$$

by Fourier transformation, where $v(z):=\sum_{k \leq n-1}\left|z_{k}\right|^{1 / \varrho_{k}}$. In fact, if $T \in$ $\Gamma^{\varrho}(\{0\})^{\prime}$, then clearly $\widehat{T}$ is in

$$
\begin{aligned}
\widetilde{H}^{\varrho}:=\left\{f \in H\left(\mathbb{C}^{n-1}\right) \mid\right. & |f(z)| \leq C_{N} \exp \left(\frac{1}{N}(v(z)+|\operatorname{Im} z|)\right) \\
& \text { for any } N \geq 1\},
\end{aligned}
$$


which coincides with $H^{\varrho}$ (this is proved by induction using Komatsu [6, Theorem 1.1]). On the other hand, if $f \in H^{\varrho}$, then there are $\left(c_{b}\right) \in \Gamma(\varrho)$ such that $f(z)=\sum c_{b} z^{b}$, hence $f=\widehat{T}$ for $T:=\sum c_{b} D^{b} \delta$.

We have also shown that $\left\{D^{b} \delta \mid b \in \mathbb{N}_{0}^{n-1}\right\}$ is total in $\Gamma^{\varrho}(\{0\})_{b}^{\prime}$, and this implies that ker $R_{2}$ is contained in $N \varrho(\{0\})$.

The most important example of a semielliptic operator is the heat operator $P(D):=\partial_{1}-\partial_{2}^{2}-\ldots-\partial_{n}^{2}\left(x_{1}\right.$ is the time variable). Then $P(x)=$ $i x_{1}+x_{2}^{2}+\ldots+x_{n}^{2}$ and $P$ is semielliptic with $\widetilde{m}=(1,2, \ldots, 2)$ and index of hypoellipticity $\varrho=(2,1, \ldots, 1)$ (see Hörmander [4, Example 11.4.15]). The canonical elementary solution for $P(D)$ is given by

$$
E(x):=\frac{1}{\left(4 \pi x_{1}\right)^{(n-1) / 2}} \exp \left(-\left|\left(x_{2}, \ldots, x_{n}\right)\right|^{2} /\left(4 x_{1}\right)\right) \quad \text { if } x_{1}>0
$$

and $E(x)=0$ if $x_{1} \leq 0$ (Hörmander [4, Theorem 3.3.3]).

Let $U$ be an open neighbourhood of 0 and let $P(D) f=0$ on $U \backslash\{0\}$. Then Theorem 2.4 leads to the following expansion: There is $g \in C_{P}^{\infty}(U)$ such that

$$
f=\left.g\right|_{U \backslash\{0\}}+\left.\sum_{b \in \mathbb{N}_{0}^{n-1}} c_{b}(f) \partial_{x^{\prime}}^{b} E(x)\right|_{U \backslash\{0\}}-\left.\sum_{b \in \mathbb{N}_{0}^{n-1}} d_{b}(f) \partial_{x^{\prime}}^{b}\left(\frac{x_{n}}{2 x_{1}} E(x)\right)\right|_{U \backslash\{0\}}
$$

where $x^{\prime}=\left(x_{1}, \ldots, x_{n-1}\right)$ and $c_{b}$ and $d_{b}$ are given by (2.3) (or the formula before $(2.5))$, i.e.,

$$
c_{b}(f):=\left(f, X_{(b, 0)}\right) \quad \text { and } \quad d_{b}:=\left(f, X_{(b, 1)}\right)
$$

and $X_{(b, j)}$ solves the Cauchy problem (2.4):

$$
\left(-\partial_{1}-\partial_{2}^{2}-\ldots-\partial_{n}^{2}\right) X_{(b, j)}=0 \quad \text { on } \mathbb{R}^{n} \quad \text { and } \quad \partial_{n}^{k} X_{(b, j)}\left(x^{\prime}, 0\right)=\frac{x^{\prime b}}{b !} \delta_{k, j} .
$$

The solutions $X_{(b, j)}$ can be calculated explicitly as follows:

$$
X_{(b, j)}=\sum_{k=0}^{|b|} Q\left(D_{x^{\prime}}\right)^{k} \frac{x^{\prime b}}{b !} \cdot \frac{x_{n}^{2 k+j}}{(2 k+j) !} \quad \text { for } j=0,1
$$

with $Q\left(D_{x^{\prime}}\right):=-\partial_{1}-\partial_{2}^{2}-\ldots-\partial_{n-1}^{2}$.

Notice that the Laurent series for $f$ vanishes for $x_{1}<0$ due to the definition of $E(x)$. This is in contrast to the case of elliptic operators and implies that $f$ is bounded on $V \cap\left\{x \mid x_{1}<0\right\}$ if $\bar{V} \subset U$. Therefore, the classification of the singularity of $f$ at 0 does not depend on the behaviour of $f(x)$ for $x_{1}<0$ in the case of the heat equation. For general hypoelliptic operators, the classification of isolated singularities will now be discussed in analogy with the corresponding results for holomorphic functions: Let $U \subset \mathbb{R}^{n}$ be a neighbourhood of zero and let $f \in C_{P}^{\infty}(U \backslash\{0\})$. We say that 
$f$ has a pole at 0 iff there are $c_{a} \in \mathbb{C}$ and $g \in C_{P}^{\infty}(U)$ such that

$$
f=\left.\left(\sum_{|a| \leq k} c_{a} \partial^{a} E+g\right)\right|_{U \backslash\{0\}} .
$$

2.5. Theorem. For $f \in C_{P}^{\infty}(U \backslash\{0\})$ the following are equivalent:

(i) $f$ can be extended to a distribution $\tilde{f}$ defined on $U$.

(ii) There is $c \geq 1$ such that

$$
|f(x)| \cdot|x|^{c} \leq c \quad \text { if } 0<|x| \leq 1 / c .
$$

(iii) There are $0 \neq y \in \mathbb{R}^{n}$ and $c \geq 1$ such that

$$
|f(x)| \cdot|\langle x, y\rangle|^{c} \leq c \quad \text { if } 0<|x| \leq 1 / c .
$$

(iv) There are $c_{a} \in \mathbb{C}$ and $g \in C_{P}^{\infty}(U)$ such that

$$
f(x)=\left.\left(\sum_{|a| \leq k, a \in I} c_{a} \partial^{a} E+g\right)\right|_{U \backslash\{0\}}
$$

where $I:=\left\{a \in \mathbb{N}_{0}^{n} \mid a_{n}<\operatorname{deg}_{x_{n}} P\right\}$.

(v) $f$ has a pole at 0 .

Proof. The implications (iv) $\Rightarrow(\mathrm{v}) \Rightarrow$ (i) and (ii) $\Rightarrow$ (iii) are trivial.

(i) $\Rightarrow$ (ii). Since $\operatorname{supp}(P(D) \widetilde{f}) \subset\{0\}$, there are $c_{a} \in \mathbb{C}$ such that

$$
P(D) \tilde{f}=\sum_{|a| \leq k} c_{a} \partial^{a} \delta
$$

Thus,

$$
\widetilde{f}=\sum c_{a} \partial^{a} E+g
$$

for some $g \in C_{P}^{\infty}(U)$. Now (ii) follows, since the estimate (2.15) is well known for $\partial^{a} E$ (see e.g. Trèves [14, Chap. 7] or Langenbruch [7, Section 2]).

(iii) $\Rightarrow$ (iv). This holds by Langenbruch [7, Satz 2.4 und Satz 1.2].

The analogue of Riemann's theorem on removable singularities reads as follows: Let $\mathfrak{B}_{P}(U)$ denote the hyperfunction zero solutions of $P(D)$ on $U$.

2.6. Theorem. Let $P(D)$ be hypoelliptic. For $f \in C_{P}^{\infty}(U \backslash\{0\})$ the following are equivalent:

(i) $f$ can be extended to $\widetilde{f} \in \mathfrak{B}_{P}(U)$.

(ii) $f$ can be extended to $\widetilde{f} \in C_{P}^{\infty}(U)$.

(iii) $\partial_{n}^{j} f$ is bounded near 0 for $0 \leq j<\operatorname{deg}_{x_{n}} P$.

Proof. (i) $\Leftrightarrow$ (ii). This holds for any partial differential operator with constant coefficients by Kaneko [5, Theorem 1].

(ii) $\Rightarrow$ (iii). This is trivial. 
(iii) $\Rightarrow$ (ii). Let $b \in \mathbb{N}_{0}^{n-1}$ and $0 \leq j<\operatorname{deg}_{x_{n}} P$. If $f$ satisfies (iii), then by Lebesgue's theorem on dominated convergence we get

$$
\begin{aligned}
\lim _{t \searrow 0} \int & \left(\partial^{(b, j)} f\left(x^{\prime}, t\right)-\partial^{(b, j)} f\left(x^{\prime},-t\right)\right) \varphi\left(x^{\prime}\right) d x^{\prime} \\
\quad & =\lim _{t \searrow 0} \int\left(\partial_{n}^{j} f\left(x^{\prime}, t\right)-\partial_{n}^{j} f(x,-t)\right)(-\partial)^{b} \varphi\left(x^{\prime}\right) d x^{\prime}=0 \quad \text { if } \varphi \in C_{0}^{\infty}\left(\mathbb{R}^{n-1}\right)
\end{aligned}
$$

since the integrand is uniformly bounded with pointwise limit equal to 0 outside $\{0\}$. Thus, the relevant distributional boundary values of $f$ all vanish, hence (ii) follows from Langenbruch [7, Satz 1.2].

The equivalence of (i) and (ii) in Theorem 2.6 answers Problem 4.4 of Ligocka [10]. Removable singularities of solutions of partial differential operators have been studied in several classes of functions by many authors (see the paper of Kaneko [5] and its references).

\section{References}

[1] G. Bengel, Das Weylsche Lemma in der Theorie der Hyperfunktionen, Math. Z. 96 (1967), 373-392.

[2] J. Bonet, R. Meise and B. A. Taylor, Whitney's extension theorem for ultradifferentiable functions, Proc. Roy. Irish Acad. Sect. A 89 (1989), 53-66.

[3] R. Harvey and J. C. Polking, A Laurent series expansion for solutions to elliptic equations, Trans. Amer. Math. Soc. 180 (1973), 407-413.

[4] L. Hörmander, The Analysis of Linear Partial Differential Operators, I, II, Grundlehren Math. Wiss. 256, 257, Springer, Berlin, 1983.

[5] A. Kaneko, On continuation of regular solutions of linear partial differential equations, in: Banach Center Publ. 27, Inst. Math., Polish Acad. Sci., Warszawa, 1992, part 1, 183-195.

[6] H. Komatsu, Ultradistributions II. The kernel theorem and ultradistributions with support in a submanifold, J. Fac. Sci. Univ. Tokyo Sect. IA Math. 24 (1977), 607628.

[7] M. Langenbruch, Randverteilungen von Nullösungen hypoelliptischer Differentialgleichungen, Manuscripta Math. 26 (1978), 17-35.

[8] -, P-Funktionale und Randwerte zu hypoelliptischen Differentialoperatoren, Math. Ann. 239 (1979), 55-74.

[9] - Surjectivity of partial differential operators on Gevrey classes and extension of regularity, Math. Nachr. 196 (1998), 103-140.

[10] E. Ligocka, On extensions of the Mittag-Leffler theorem, Ann. Polon. Math. 68 (1998), 249-256.

[11] R. Meise und D. Vogt, Einführung in die Funktionalanalysis, Vieweg, Braunschweig, 1992.

[12] N. N. Tarkhanov, Laurent expansions and local properties of solutions of elliptic systems, Sibirsk. Mat. Zh. 29 (1988), no. 6, 124-134 (in Russian).

[13] —, The Analysis of Solutions of Elliptic Equations, Kluwer, Dordrecht, 1997.

[14] F. Trèves, Linear Partial Differential Equations with Constant Coefficients, Gordon and Breach, New York, 1966. 
[15] M. Wachman, Generalized Laurent series for singular solutions of elliptic partial differential equations, Proc. Amer. Math. Soc. 15 (1964), 101-108.

Department of Mathematics

University of Oldenburg

D-26111 Oldenburg, Germany

E-mail: langenbruch@mathematik.uni-oldenburg.de

Received on 9.6.2001;

revised version on 8.11.2001 